\title{
Stirling to Flight Initiative
}

\author{
Kenneth E. Hibbard ${ }^{\dagger}$ (Kenneth.Hibbard@jhuapl.edu, 443-778-1458), Lee S. Mason* \\ (Lee.S.Mason@nasa.gov), Obi Ndu ${ }^{\dagger}$ (Obibobi.Ndu@jhuapl.edu), Clayton Smith ${ }^{\dagger}$ \\ (Clay.Smith@jhuapl.edu), James P. Withrow* (James.P.Withrow@nasa.gov), \\ ${ }^{\dagger}$ Applied Physics Laboratory \\ Johns Hopkins University \\ 11100 Johns Hopkins Rd. \\ Laurel, MD 20723 \\ " Glenn Research Center \\ National Aeronautics and Space Administration \\ 21000 Brookpark Road \\ Cleveland, OH 44135
}

\begin{abstract}
NASA has a consistent need for radioisotope power systems (RPS) to enable robotic scientific missions for planetary exploration that has been present for over four decades and will continue into the foreseeable future, as documented in the most recent Planetary Science Decadal Study Report. As RPS have evolved throughout the years, there has also grown a desire for more efficient power systems, allowing NASA to serve as good stewards of the limited plutonium-238 $\left({ }^{238} \mathrm{Pu}\right)$, while also supporting the ever-present need to minimize mass and potential impacts to the desired science measurements. In fact, the recent Nuclear Power Assessment Study (NPAS) released in April 2015 resulted in several key conclusion regarding RPS, including affirmation that RPS will be necessary well into the 2030s (at least) and that ${ }^{238} \mathrm{Pu}$ is indeed a precious resource requiring efficient utilization and preservation.
\end{abstract}

Stirling Radioisotope Generators (SRGs) combine a Stirling cycle engine powered by a radioisotope heater unit into a single generator system. Stirling engine technology has been under development at NASA Glenn Research Center (GRC) in partnership with the Department of Energy (DOE) since the 1970's. The most recent design, the ${ }^{238} \mathrm{Pu}$-fueled Advanced Stirling Radioisotope Generator (ASRG), was offered as part of the NASA Discovery 2010 Announcement of Opportunity (AO). The Step-2 selections for this AO included two ASRGenabled concepts, the Titan Mare Explorer (TiME) and the Comet Hopper (CHopper), although the only non-nuclear concept, InSight, was ultimately chosen. The DOE's ASRG contract was terminated in 2013. Given that SRGs utilize significantly less ${ }^{238} \mathrm{Pu}$ than traditional Radioisotope Thermoelectric Generators (RTGs) - approximately one quarter of the nuclear fuel, to produce similar electrical power output - they provide a technology worthy of consideration for meeting the aforementioned NASA objectives.

NASA's RPS Program Office has recently investigated a new Stirling to Flight (S2F) initiative with the objective of developing a 100-500 $W_{e}$ Stirling generator system. Additionally, a different approach is being devised for this initiative to avoid pitfalls of the past, and apply lessons learned from the recent ASRG experience. Two key aspects of this initiative are a Stirling System Technology Maturation Effort, and a Surrogate Mission Team (SMT) intended to provide clear mission pull and requirements context. The S2F project seeks to lead directly into a DOE flight system development of a new SRG. This paper will detail the proposed S2F initiative, and provide specifics on the key efforts designed to pave a forward path for bringing Stirling technology to flight.

\section{TABLE OF Contents}

1. INTRODUCTION ..................................................1

2. S2F OBJECTIVES .............................................2

3. S2F APPROACH..........................................3

4. SuRrogate Mission TEAM (SMT)................4

5. STIRling TeChNOLOGY MATURATION AND

DEVELOPMENT .5

6. RISK INFORMED LIFETIME MODELING AND

TESTING .......................................................66

7. CONCLUSION......................................................8

REFERENCES......................................................99

BIOGRAPHIES................................................99

\section{INTRODUCTION}

A recent Nuclear Power Assessment Study (NPAS) [1] was completed in 2015, under the guidance of NASA's Radioisotope Power Systems (RPS) Program Office. The principal objectives of this study were to discuss a sustainable strategy and examine the current capabilities for providing safe, reliable, and affordable nuclear power systems to enable NASA Science Mission Directorate (SMD) missions and their extensibility to Human Exploration and Operations Mission Directorate (HEOMD) needs over the next twenty years.

NASA's need for RPS to enable robotic scientific missions for planetary exploration has been a "given" for over four decades, and continues to remain an ongoing need based on the findings of the most recent Planetary Decadal Survey of 2011, "Vision and Voyages" [2]. NPAS goes on to conclude that this need resides primarily within planetary science, with a few potential exceptions that support Heliophysics Science Division, such as the Interstellar Probe concept contained within the Heliophysics Decadal Survey of 2013: "Solar and Space Physics: A Science for a Technological Society" [3]. This need for planetary science is expected to continue well into the 2030's, most likely longer. 
While future human missions, such as a surface mission to Mars, will likely require some form of nuclear power, these efforts are forecast to need 35 to $40 \mathrm{~kW}_{\mathrm{e}}$ which is not reasonable with current, or envisioned, RPS technology. The HEOMD missions are likely to call for fission-based nuclear power systems that rely on enriched ${ }^{235} \mathrm{U}$ rather than ${ }^{238} \mathrm{Pu}$. There may be some commonality in the technologies used, such as within Stirling converters, but is considered beyond the scope of the current proposed charter for a SRG development activity.

Lastly, the NPAS report concluded that single planetary science mission power requirements are unlikely to exceed $\sim 600 \mathrm{~W}_{\mathrm{e}}$, and that this required power level is more efficiently fulfilled with an RPS than a fission power system (FPS). For future RPS-powered missions to utilize greater electrical power than today $(\sim 110-125 \mathrm{~W}$ per generator) requires increased ${ }^{238} \mathrm{Pu}$ production and/or flight qualification of higher efficiency converters, for example thermoelectric enhanced multi-mission radioisotope thermal generator (eMMRTG) and/or dynamic Stirling converters.

The new Stirling to Flight (S2F) initiative seeks to address several of the key NPAS findings, specifically targeting the needs of planetary, robotic exploration missions through the 2030 s. It hopes to initially produce an SRG with roughly equivalent electrical output using less ${ }^{238} \mathrm{Pu}$ than today's flight-ready systems by employing dynamic Stirling converter technology. One of the principal lessons from past efforts is to execute the S2F as a flight system development project, rather than as a technology development effort, driving the design and testing of a system to be used in flight. It is really the integration of two major efforts that culminate in a DOE contract for a SRG flight system development project. These two focus areas are 1) establishment of a Surrogate Mission Team (SMT) that provides clear mission pull and requirements context, as described in Section 4 below, and 2) a Stirling technology maturation effort, detailed in Section 5, both supported by Risk Informed Lifetime Modeling described in Section 6.

\section{S2F OBJECTIVES}

The new S2F initiative was drafted in Spring 2015. The objectives of the S2F initiative are:

- Develop a 100-500 $\mathrm{W}_{\mathrm{e}}$ Stirling generator RPS for integration onto a mission launch opportunity no earlier than 2028 that is robust, manufacturable, and reliable (fault-tolerant, long-life) with reasonable life-cycle and sustainability costs

- The final version of this system should produce higherpower output per volume of ${ }^{238} \mathrm{Pu}$ consumed compared to current RPS such as the MMRTG (e.g., $\sim 125 \mathrm{~W}_{\mathrm{e}}$ BOL using only 2 GPHS vice the MMRTG's 8 GPHS)

- This SRG should be constrained to a reasonable mass, $<50 \mathrm{~kg}$ per unit, and seek to be as mass efficient as possible
- Any new system should also be designed to be modular, allowing for the use of 1-4 generators per mission

- The SRG should limit waste heat (i.e., target $\sim 500 \mathrm{~W}_{\text {th }}$ per unit) that could negatively impact key science measurements being made

Additionally, the $\mathrm{S} 2 \mathrm{~F}$ initiative seeks to invoke lessons learned from the previous Advanced Stirling Radioisotope Generator (ASRG) efforts, with greater consideration for robustness, manufacturing, and reliability.

Considering the first objective, one immediately seeks clarification on exactly what is meant by "robust, manufacturable, and reliable with reasonable life-cycle and sustainability costs." These terms need to be clearly defined and quantified into requirements that will drive the system design. Defining these requirements is part of the tasks in the early S2F development phases.

Another key objective of the S2F effort is to actually demonstrate the technology in flight. To this end, the team is focusing on the first flight of a Stirling converter, keeping the expectations and requirements on this technology modest. It is anticipated that there will be SRG design evolutions for future flights beyond the initial one, that can build upon the "to-be proven" technology, focusing on typical engineering enhancements. Therefore, the initial SRG is only seeking to provide $100-500 \mathrm{~W}_{\mathrm{e}}$, and does not

\section{Technology Selection and Process to Flight}

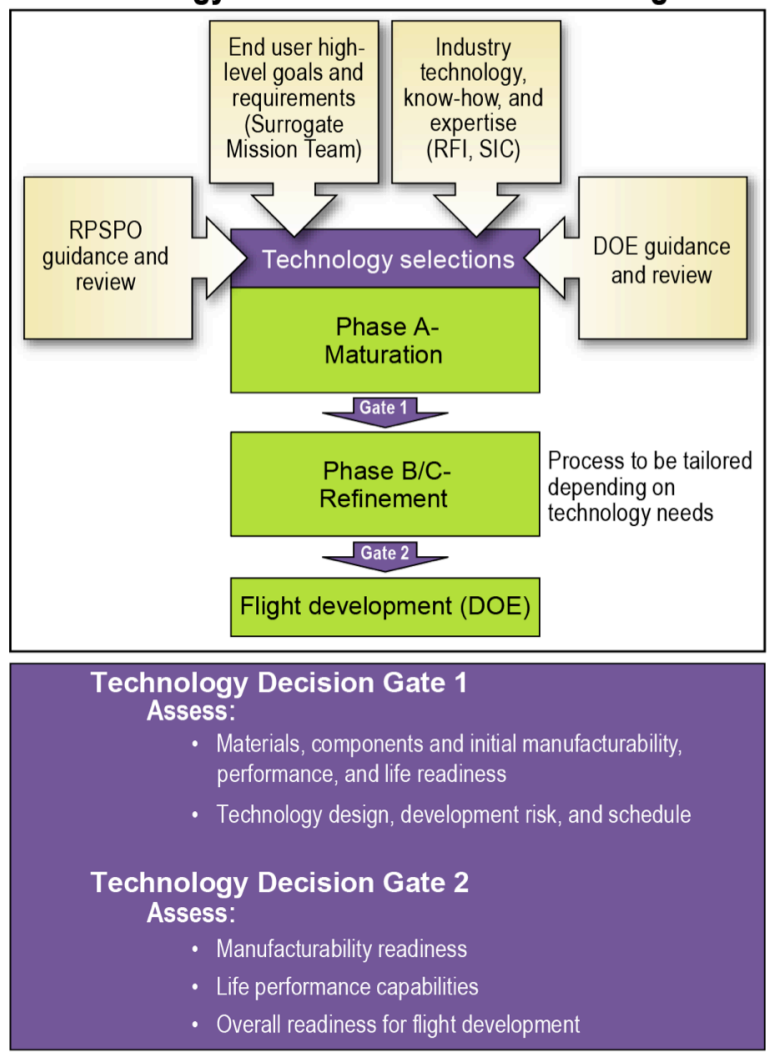

Figure 1. Technology selection and process to flight builds upon successful MMRTG implementation. 
have a requirement to maximize efficiency but rather to focus on converter technology that is reliable, predictable, and repeatable.

\section{S2F APPROACH}

The S2F approach is to build upon successful technology maturation methodologies from the past, especially those involving interactions with the Department of Energy (DOE) on nuclear powered systems. Specifically, S2F intends to leverage the MMRTG and eMMRTG technology Maturation Operational and Evaluation Model as shown in Figure 1.

The S2F initiative is envisioned to employ a four-phase implementation approach, as shown in Figure 2. Phase 1, Preparation and Discovery, focuses on the establishment of both a Management Team (MT) and the Surrogate Mission Team (SMT), releasing a Stirling RFI and analyzing the results, initial $\mathrm{S} 2 \mathrm{~F}$ requirements development, and identification of the acquisition strategy.

The MT oversees both the Stirling technology development and the SMT. The MT will ensure and facilitate coordination between the NASA and DOE agencies, and across all of the RPS program elements. This team consists of program managers from RPS and DOE, the RPS Program Planning and Assessment Manager, the Stirling Cycle Technology Development Project Manager, and both the NASA and DOE contract officers. The major products that this team is responsible for include the Stirling request for information (RFI), the S2F acquisition strategy, InterAgency Agreements (IAA), gate review criteria and board membership consistent with NASA Procedural Requirements 7123 (or DOE equivalent), and the top-level integrated budget and schedule.

Fundamentally, as its name suggests, the SMT functions as a surrogate flight mission team until NASA selects an actual flight mission, and serves as the requirements and technical authority during S2F. This team includes leadership from the NASA RPS Office and the DOE, power systems expertise from Glenn Research Center (GRC), and representatives from each of the major robotic mission flight implementers utilized by NASA, specifically the Johns Hopkins University Applied Physics Laboratory (JHU-APL or APL), the Jet Propulsion Laboratory (JPL), and NASA Goddard Space Flight Center (GSFC). Additional details on the SMT are provided in Section 4 below.

The Preparation and Discovery Phase proceeds to gather information, scope out requirements, and identify the acquisition strategy. NASA released an RFI in 2015 to assess Stirling technology options available and how they can be incorporated into the desired space qualified power system. The goal was to understand Stirling industrial suppliers and their demonstrated capabilities (including precision flight hardware manufacturing ability), for both converter providers and system integrators; potential partnership approaches between different organizations were also identified. The RFI sought information on SRG derivative and scalability potential, as well as an understanding of any intellectual property concerns and potential licensing opportunities. The responses were analyzed and assessed by a joint NASA-DOE review team, a subset of which traveled to the various vendor locations

\section{S2F Notional Schedule to Support NF Type Mission}

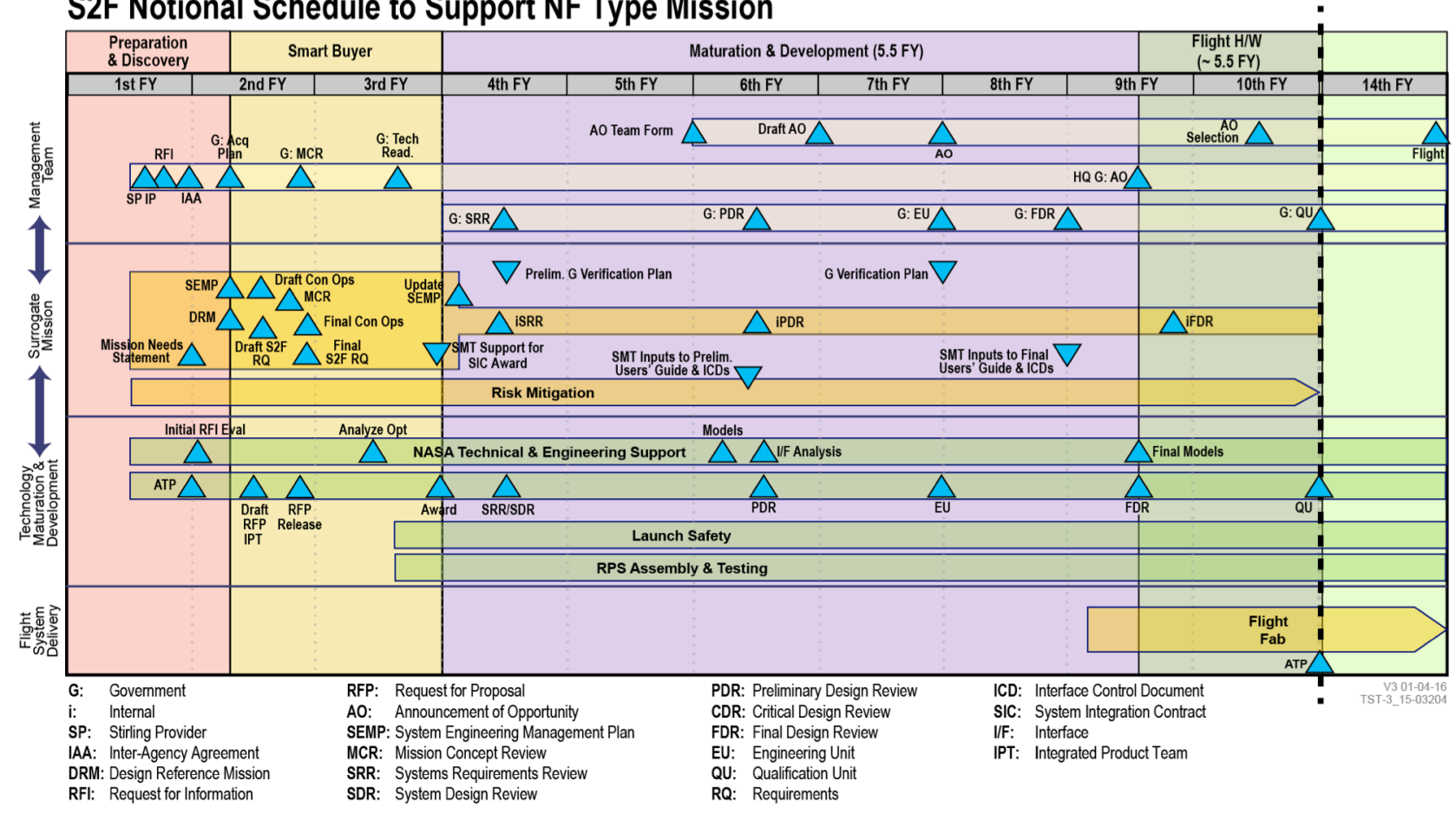

Figure 2. The S2F phased development fosters technology development driven by flight mission centers' needs and concerns, targeting a New Frontiers class mission with a potential launch in 2028. 
for targeted, more in-depth investigation.

An initial set of requirements is being defined by the SMT using a traditional systems engineering process, working from a mission context that includes as many potential SRG-enabled missions as realistic, defining mission-level requirements, and working down to flight-system, subsystem, and (if appropriate) unit-level requirements. A notional concept of operations is being derived in parallel with the context missions and the initial requirements set.

To date, all of the milestones identified in the Preparation and Discovery Phase have been achieved as scheduled. The details of how S2F will advance into the Smart Buyer Phase remain in negotiations with NASA Headquarters, with its notional start at least six months into the future at the time of this paper.

Phase 2, Smart Buyer, is intended to ensure that the best available Stirling technologies are evaluated and selected to ultimately move into development and flight fabrication. The S2F initiative realizes that the true technology development effort is contained within the Stirling converter. During this period, it is anticipated that several different converters will be tested, with multiple providers possibly receiving funding to provide engineering or demonstration test units. Think of Phase 2 as a test-drive for the Stirling converters where different drivers get a chance to kick the tires and decide which models they are favoring. This phase will include a technology readiness assessment as a gate review by the government. In addition, during this phase the MT will finalize the SRG acquisition strategy, and the DOE should release a draft Request for Proposal (RFP) that addresses both converter providers and SRG system integrators. The SMT will work to update the mission and system requirements that will flow down to the DOE to drive their RFP. The Smart Buyer phase concludes with the DOE awarding a Stirling technology maturation system contract to specifically develop the next SRG.

The third phase will mature and develop the SRG. This phase is envisioned to provide greater than five years for a typical complex system design, fabrication, and verification process. This phase focuses on SRG development moving through the tradition formal implementation gates including Systems Requirements Review (SRR)/System Design Review (SDR), Preliminary Design Review (PDR), and a Final Design Review (FDR); refer to Figure 2 for relative timing. Prior to the FDR, an SRG engineering unit must be provided and fully tested (exact test definition and success criteria are TBD at present) as another formal gate. To establish confidence in SRG availability with potential users, it is strongly recommended that the FDR must be successfully completed prior to NASA offering an SRG in any future AOs. The SRG maturation and development phase ends once a fueled qualification unit has successfully gone through system validation.

The DOE will hold the contract for a Stirling generator provider/integrator, who may subcontract to a government- specified Stirling converter provider as determined during the previous phase. There may also be an Integrated Systems Engineering Team (ISET) formed to guide and advise the SRG development. This ISET is expected to consist of both the SMT and a systems engineering team from the contractors, along with any independent technical experts that may be deemed necessary for success by the MT. The ISET utilizes the SMT to provide the mission endusers' perspective, and mission pull, as the SRG moves through development, and the inevitable design evolution as the technology is matured. This intent is to maximize the design space the contractors have to work with, while ensuring all of the mission requirements are met or negotiated to facilitate technical readiness, robustness, and reliability. The ISET and MT will hold internal project reviews to help ensure success, or to suggest corrective actions when necessary prior to the formal gate reviews. Note, it is possible that the ISET could also be created in the previous phase to help define the SRG and its development plan, but would need to be done in a manner that does not restrict potential respondents to the DOE RFP process.

The fourth and final phase is the actual SRG flight system fabrication and delivery. This is when the DOE-contracted SRG provider takes the system design proven by the engineering and qualification units and produces the final flight hardware that will be delivered to NASA for use on a flight mission. It is expected that this phase will be in parallel to a New Frontiers class competitive AO from NASA, and timed so that the final SRG is delivered to Kennedy Space Center in time for the flight mission's integration and test flow requirements.

\section{Surrogate Mission Team (SMT)}

The SMT was formed at the start of the S2F initiative and provides flight mission perspective, involvement, and continuity throughout the various phases described earlier; the SMT empowers the S2F initiative to be executed as a "flight" development rather than as a pure technology endeavor. The SMT serves as the requirements technical authority during $S 2 F$. Once an actual mission is awarded in the final flight hardware delivery phase, the SMT will transition their products and knowledge to that actual mission team. From that point forward, it is expected that continued efforts will focus exclusively on the implementation and execution of the chosen flight project. SMT personnel will most likely continue to support the RPS Program Office as SRG Integration Lead and consultants, in much the same manner that JPL personnel have done with RTGs in the past.

Core to this approach is the formation of a crossorganizational team, with all of the key stakeholders and agency leadership engaged. This team includes leadership from the RPS Program Office and from the DOE due to the nuclear fuel used to provide the input heat that drives power generation. GRC is involved as the lead center for NASA power systems technology development. Applying a lesson learned from the ASRG project, the leading NASA mission 
implementation centers of the APL, JPL, and GSFC are all key participants. Inclusion of the mission centers is an essential component, as these organizations provide the necessary mission pull and end-user perspective, and context, needed for S2F to be successful. These organizations also carry forth their expertise in bringing systems successfully to flight, and by having all three involved, ensure that the developed system is appropriately defined, matured, and will meet mission requirements; working to get buy-in from all three of the mission centers fosters development of a suitable system that will meet flight mission needs within acceptable risk.

The SMT's principal charter is to ensure that mission requirements, trades, and risks are completely integrated throughout the S2F technology development and maturation. Their key functions are essential to success of the early S2F initiative phases when their major deliverables are generated. The primary deliverables from the SMT include a System Engineering Management Plan (SEMP) to guide its implementation, a mission needs statement to support the DOE's processes for interacting with NASA, mission context (i.e., design reference mission), concept of operations, and S2F requirements. The SMT also oversees the definition and implementation of the risk informed lifetime testing and modeling activities, as detailed in Section 5.

The SMT will provide risk management and mitigation support throughout the SRG initiative, bringing forth the unique perspective that only a flight mission implementer, and final SRG end-user, can provide. The risk management approach is consistent with existing process at the mission implementation centers, and compliant with NASA Procedural Requirements 8000.4.

\section{Stirling TeChnology MAturation AND DEVELOPMENT}

The previous effort to develop the ASRG is well documented with references to system design [4], system testing [5], and Stirling converter maturation [6]. After the ASRG flight contract was terminated in 2013, GRC continued development of the final engineering model Advanced Stirling Converters (ASCs; designated ASC-E3)

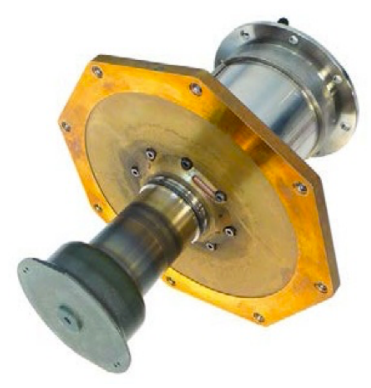

Figure 3. ASC-E3 at Sunpower Inc., shown in Figure 3. The ASC development has produced a total of 29 converters with over 400,000 hours of cumulative operation at GRC.

The ASC-E3 effort has resulted in eight converters, with the latter four units built using flight-approved processes and assembled in a Sunpower cleanroom. All but the last unit have been delivered to GRC for independent testing and verification.

The first two units (\#1 and \#2) were operated at GRC as stand-alone units for extended performance mapping (approximately $3500 \mathrm{hrs}$.) and then integrated into the Engineering Unit \#2 (EU2) system, shown in Figure 4. The EU2 test ended prematurely in January 2015 due to converter power fluctuations. The investigation to determine root cause is still on-going with four leading theories: 1) internal collisions of moving parts, 2) adverse interactions from the pulse-width-modulated electrical controller, 3) insufficient running clearances on moving components, and 4) excessive forces imparted on converters from the external housing structure. Recommendations are being prepared by the investigation team to update processes and/or revise the design to avoid these issues.

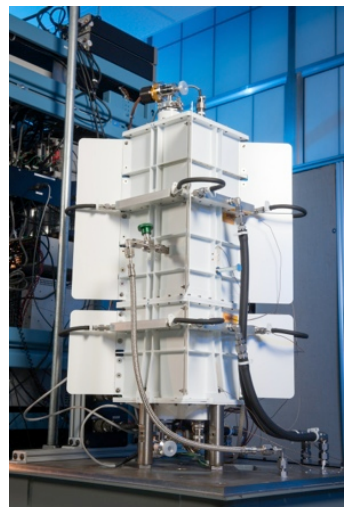

Figure 4. EU2 Generator Housing Assembly

One E3 converter (\#7) experienced operational issues during production before it was delivered to GRC. The unit was found to have significant internal fiber debris from an improperly installed regenerator that disrupted operation. Regenerator debris can present a problem in piston gap clearances and at the internal gas bearing check valve. The \#7 converter was eventually scrapped allowing detailed sectioning of the components to determine root cause. As a result, Sunpower was directed to produce E3 \#9, planned for delivery in December 2015. The investigation resulted in a minor design change to the regenerator, and revised procedures for installation. Future designs that eliminate debris or increase the converter's tolerance to debris would be beneficial.

The other E3 converters are still being tested at GRC with the goal of subjecting the converters to a typical mission lifecycle. The first step after delivery of the converters from Sunpower is to map their performance against the design specification. Testing is performed on individual units using an electrical heat source simulating the ${ }^{238} \mathrm{Pu}$ General 
Purpose Heat Source (GPHS) module. The mapping includes steady-state operation at seven distinct operating conditions varying heat input, acceptor temperature, and rejection temperature. Afterwards, the converters are processed under conditions simulating the major phases of a flight mission including system integration/fueling (where the two Stirling converters are installed as a dual, opposed assembly), dynamic launch environments (where the operating units are subjected to launch vibrations), and longduration mission cruise (where the units are operated 24/7 for extended duration).

Prior to the ASC, NASA and DOE were developing the SRG-110 which used Technology Demonstration Converters (TDC) built by Infinia Corporation [7]. These units use internal flexure bearings, rather than the hydrostatic gas bearings used in ASCs. A total of 16 TDCs were produced in the late 1990s and early 2000s with 4 units, shown in Figure 5, still in 24/7 operation at GRC with one pair at 84,000 hours and the other at 95,000 hours of run time.

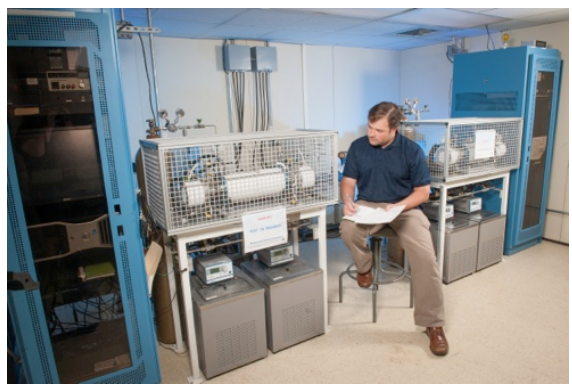

Figure 5. TDC Test Stands at GRC

In parallel with the ASRG flight system development, lower technology readiness level (TRL) research was also being performed at GRC [8]. The low TRL research focused on advanced components and alternative system implementation approaches. These technologies are candidates for inclusion in the next SRG should their development continue to a successful conclusion. At the Stirling hot-end, research was performed to improve the thermal insulation and identify possible methods to divert the GPHS heat from the converters to allow shutdown and restart after fueling. A multi-layer insulation (MLI) package was built and tested with a Stirling thermal mockup demonstrating a lower mass, higher efficiency alternative to the bulk, solid insulation used in ASRG. To demonstrate backup heat source cooling, a variable conductance heat pipe was integrated with an operating Stirling converter and an electric heat source. A test, performed in May 2015, verified that the heat pipe could automatically redirect the heat to a high temperature radiator during Stirling converter stoppages without violating temperature limits. Once the Stirling converter was restarted, the heat pipe automatically restored heat flow to the converter.

At the Stirling cold-end, research was performed to extend the upper temperature range of the Stirling alternator, and replace the conductive heat rejection interface with a titanium-water heat pipe. A modified ASC alternator using high temperature magnets was built and operated, while testing continued on high temperature organic materials for use in the converter. The elevated alternator temperature enables Venus flyby maneuvers as well as lunar and Mars operation at the maximum sink temperature. To improve heat rejection and enable scaling to higher power, a flat plate heat pipe was developed as a direct replacement for the ASRG cold side adapter flange. In July 2015, its performance was demonstrated under both hyper-g and micro-g environments on a Black Brant IX sounding rocket.

Other tasks in process under the low TRL research effort include dynamic balancers to allow single converters to operate with low vibration, and an alternative electrical controller, developed by APL, with improved fault management. The APL controller was coupled with a pair of early model ASCs at GRC's RPS System Integration Lab in September 2014 to demonstrate stable operation with a spacecraft bus and representative electrical loads [9].

The Stirling development activities at GRC have been reformulated under the new Stirling Cycle Technology Development Project with the goal of continuing work on systems, converters, controllers, testing, and research. The RPS Program recently released an industry Request for Information (RFI) seeking new approaches for Stirling technology, as described in Section 3 above. The objective is to identify robust and reliable Stirling converter designs that deliver high efficiency, low mass, simple operation and long life (without maintenance) for deep-space missions lasting 10 years or more. Ideally, this process will yield competing converter options that can be matured in parallel prior to a system RFP by DOE.

\section{RISK INFORMED LIFETIME MODELING AND TESTING}

A critical success factor in meeting the goals of the S2F Initiative is demonstrating the viability of the RPS technology and its likelihood of performing as expected for as long as needed. Reliability estimation is part of the approach for lifetime analysis; however traditional reliability analysis for lifetime prediction has inherent challenges that complicate the process especially for systems with relatively long lifetime requirements.

The dilemma for demonstrating reliability or service life for items that must last a long time (perhaps over a decade) is that the testing is expensive, time consuming, must satisfy multiple sets of expectations, and address a variety of requirements simultaneously. Although frequently used as decision tools for planning and designing system test campaigns, traditional statistical methods for sample size determination and test duration simply do not yield results with enough confidence to adequately reduce the technology maturation risk associated with RPS solutions with long lifetime requirements. For example, if a program requires a reliability of 0.90 at 15 years $(130,000$ hours) with a confidence of $90 \%$, the number of hours needed for demonstration with no failures is 2.9 million hours using sample theory with an exponential distribution assumption 
[10]. Even turning to state-of-the-art Bayesian methods such as the WeiBayes Zero-Failure formula [11] yields 1.2 million hours of testing. These hours increase tremendously if failures have to be taken into account.

To balance the challenge of time and resource limitations, a Risk-informed Life Testing approach has been adopted for guiding the testing and risk-reduction activities envisioned for the S2F Initiative. Rather than focus exclusively on how to demonstrate compliance with reliability requirements, the Risk Informed Life Testing process [12] addresses integrating test and performance data of heritage components and physics-of-failure models for new technology into a comprehensive framework. The result of the process includes reduction of uncertainty of the results through targeted testing of limited data components and a framework for using data from all relevant sources.

Part of the Risk-informed Life Test process is a thorough review of existing heritage SRG reliability, physics-offailure, and lifetime models in addition to the performance and test evidence collected to support such models. With an understanding of the reliability and lifetime implications for a new Stirling technology concept, the following steps are to be implemented:

(1) Establish the system lifetime goal

(2) Develop physics-based models to analyze the system and determine the probability of meeting that goal and the associated uncertainty or margin

(3) Identify data needed to support models and determine sources for data: in-house, system heritage, or new testing

(4) Analyze data and update probability predictions

Within NASA programs, lifetime demonstration also involves testing for many hours. In contrast, the Riskinformed Life Test framework views testing as a targeted opportunity to reduce uncertainty in life models. While most performance attributes or parameters such as temperature, speed, voltage, or material strength contain deterministic characteristics, verification by analysis must play a key role when assessing systems with long mission times. The idea is to develop high confidence in these models instead of a life test that is equivalent to decades of operation. Material

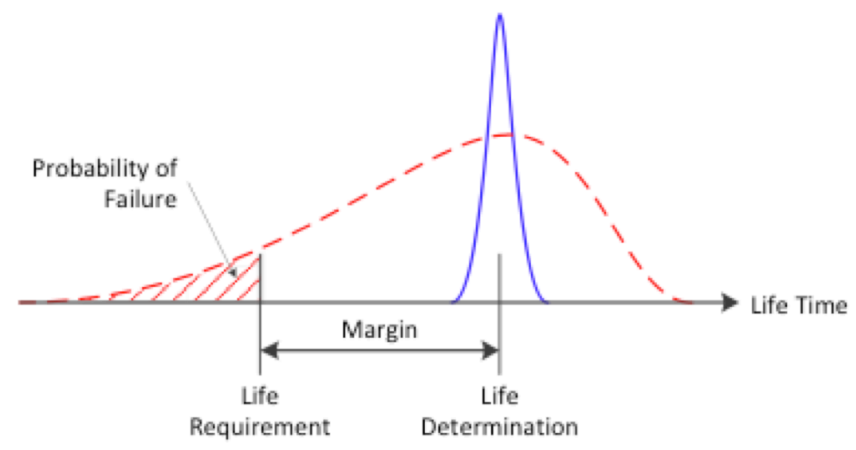

Figure 6. The Probabilistic Nature of Margins

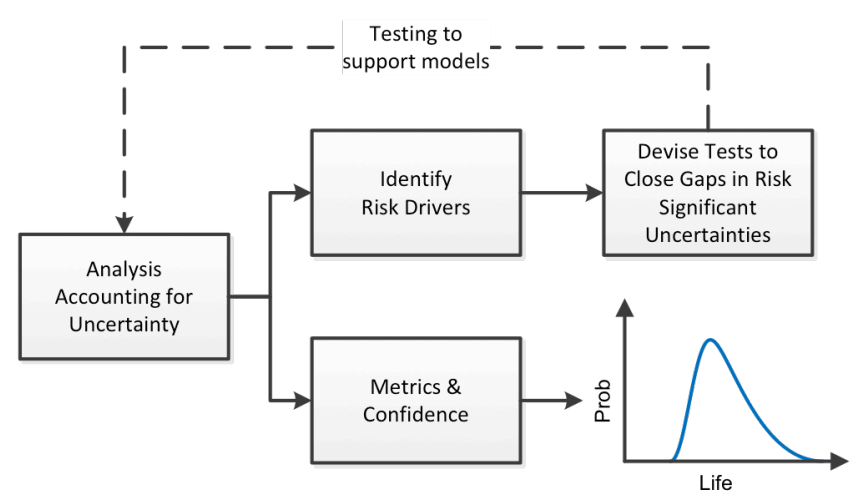

Figure 7. Risk Informed Test Planning Process

properties, for example, are characterized through laboratory testing and their physical properties used to predict performance over the operating years imposed on the project. If the end of life performance margins are at least as large as those quantified for the previous missions and the confidence in these margins is at least as great as the confidence in the previous margin estimates (see Figure 6), then the new technology will have the same probability and confidence that it will complete its mission successfully.

Through this integrated analysis and testing process the program indicates to the community the confidence it has in meeting lifetime and reliability goals, taking the form of a probability or margin. The analysis examines the system for all potential threats to not meeting the performance requirements for the period specified. As illustrated in Figure 7, the testing supports the analysis in an iterative manner to reduce the uncertainties. Life metrics are computed along with the confidence in those metrics.

The concept SRG system is decomposed into subsystems and components and logic developed as a risk prediction model. Failure modes, failure mechanisms, tight margins, and environmental interactions are all identified which can potentially cause failure. A variety of reliability engineering methods will be used, including, but not limited to: Failure Mode and Effects Analysis (FMEA), Worse-Case Analysis, or Physics of Failure analysis. The results are combined into the risk model where the postulation of failure scenarios is formed. Data is gathered from a variety of sources (previous flight programs, similar systems, industry databases, test results, and engineering judgment). All available data is used (although some are more relevant than others) to fully develop the understanding of the system. The models are then exercised to identify the significant risk events and to determine the probability of meeting the life requirement. Figure 8 shows the realization (top box) that the SRG system is a mixture of "old" technologies with a particular usage history and "new" technologies with perhaps only a test history. A risk model ties all subsystems together in a framework that models the failure behavior of the system.

Tests are designed to exercise elements of the system where there is limited understanding or relevant heritage data. 


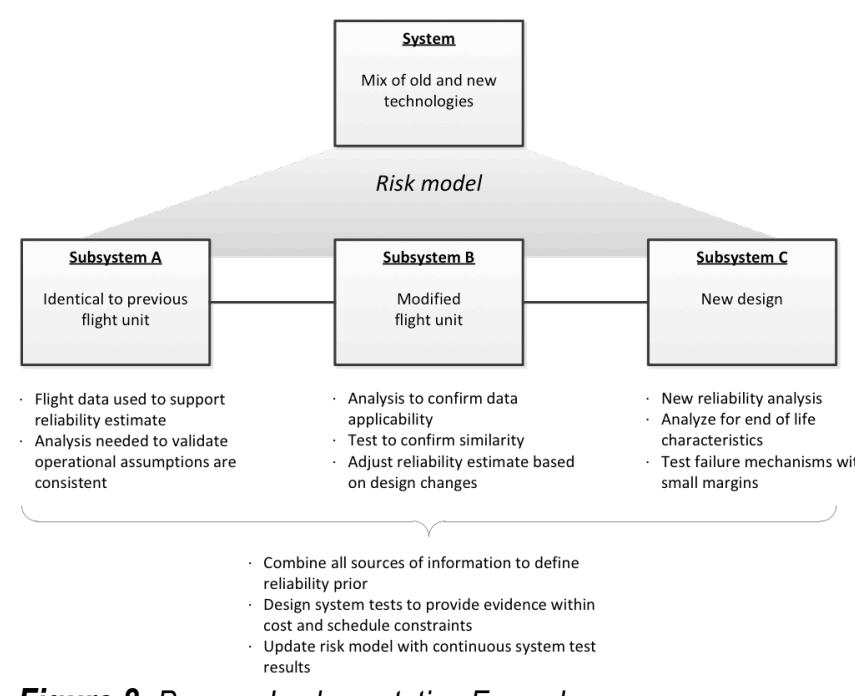

Figure 8. Process Implementation Example

These tests are targeted to better define the failure models and improve the quality of the data supporting the models. This test definition and design approach changes the paradigm from testing a system for many hours to show confidence in the lifetime, to increasing confidence in the understanding of how the system is likely to fail at end of life. The resultant test data is incorporated into the models for the concept SRG. In the parlance of Bayesian analysis, the models are updated to reflect the effect of observed relevant evidence. From these results the design can change to accommodate and mitigate the failure modes.

Multi-level data will be used as input into a system risk model (fault tree, reliability block diagram, or PRA). The resultant metric for each subsystem is an estimate of the reliability and an uncertainty distribution, built on relevant information available on the subsystem. An estimate of the probability of failure is made and becomes the system level prior, meaning the initial estimated distribution. System level testing is then designed and implemented (within cost and schedule constraints) with the results being the evidence needed to update the System Risk Model. The result is the probability of meeting the life requirement with a measure of the confidence in that metric. As more time and data are gathered, the credibility of the metrics shifts accordingly. Instead of defining a large test program and making no statements about its reliability until the test program is completed, in this scheme, the confidence is based on performance data, test data, and analysis.

Since, the data variability and model uncertainty is inherent in the updated models, the confidence about the probability can also be calculated. In addition, this quantitative approach allows the designers to see the sensitivity (and therefore cost-benefit) of performing certain tests or modifying design elements or materials.
Using risk informed processes to demonstrate compliance with life requirements (e.g., demonstrating a certain reliability will be achieved with a specified level of confidence) is a useful technique for minimizing resources (e.g., budget and schedule) needed for developing new technologies. Moreover, using risk informed processes to establish life requirements, by directly trading reliability, confidence, and technology performance with resources, enhances the ability of projects and stakeholders to develop life requirements compatible with expectations and resource constraints

\section{Conclusion}

The S2F initiative seeks to provide the roadmap and framework for the development of an SRG for use in future planetary science space missions. This effort has been scoped to focus on the first flight SRG units, concentrating on maturing the technology, and assuming subsequent design iterations will serve to optimize and improve performance. This effort looks to build upon past programs, such as the SRG-110 and ASRG, and incorporate lessons learned while targeting a no-earlier-than 2028 launch opportunity.

One of the principal lessons is to execute the S2F effort as a flight system development project, rather than as a technology development effort, driving the design and testing of a system to be used in flight. Core to this undertaking is the use of the SMT to provide mission pull, end-user perspective, and an honest level of buy-in from the primary NASA robotic mission implementation centers.

All of the recent testing and evaluations from past Stirling programs, combined with current assessments of potential providers, will be incorporated into a smart-buyer approach to attack the real technology risk area, specifically the Stirling converters. S2F looks to reduce the risk and mature these converters prior to engaging a system integrator that will, under contract to the DOE, develop the eventual SRG system.

Another primary objective of the S2F Initiative is to utilize risk informed lifetime modeling and testing to demonstrate compliance with life requirements while balancing time and resource allocations. These techniques can help solve the challenges of verifying performance and reliability for longlife ( $>10$ years) components without necessitating equally long-lived prelaunch testing in simulated environments, and provide inputs for improved decision making throughout the development process. 


\section{REFERENCES}

[1] R.L. McNutt, Jr., S.M. Aleman, M.J. Amato, W.P. Carroll, L.A. Dudzinski, J. McKamy, C. Moore, C. Reed, K.R. Reh, J.A. Sholtis, Jr., R.A. Stephen, Nuclear Power Assessment Study - Final Report, Johns Hopkins University Applied Physics Laboratory, Laurel, MD, 2015.

[2] National Research Council, Vision and Voyages for Planetary Science in the Decade 2013-2022, National Academies Press, Washington, D.C., 2011.

[3] National Research Council, Solar and Space Physics: A Science for a Technological Society National Academies Press, Washington, D.C., 2013.

[4] Chan, J., Dennis, H., Hoye, T., and Leland, D., "Development of Advanced Stirling Radioisotope Generator For Planetary Surface and Deep Space Missions", Proceedings of the 6th International Energy Conversion Engineering Conference (IECEC), AIAA-2008-5768, Cleveland, OH, 2008.

[5] Lewandowski, E., Oriti, S., and Schifer, N., "Characterization of the Advanced Stirling Radioisotope Generator EU2," Proceedings of the 13th International Energy Conversion Engineering Conference (IECEC), AIAA-2015-3808, Orlando, FL, 2015.

[6] Wong, W., Wilson, S., Collins, J., and Wilson, K., "Advanced Stirling Converter (ASC) Technology Maturation," Proceedings of the 13th International Energy Conversion Engineering Conference (IECEC), AIAA-20153806, Orlando, FL, 2015.

[7] Schreiber, J. and Thieme, L., "Final Results for the GRC Supporting Technology Development Project for the 110Watt Stirling Radioisotope Generator (SRG110)," NASA/TM-2007-214839, June 2007.

[8] Wilson, S., Schifer, N., Williams, Z., and Metscher, J., "Overview of Stirling Technology Research at NASA Glenn Research Center," Proceedings of the 13th International Energy Conversion Engineering Conference (IECEC), AIAA-2015-3905, Orlando, FL, 2015.

[9] Dugala, G., Taylor, L., Bell, M., Dolce, J., Fraeman, M., and Frankford, D., "Advanced Stirling Converter Dual Converter Controller Testing at NASA Glenn Research Center in the Radioisotope Power Systems System Integration Laboratory," Proceedings of the 13th International Energy Conversion Engineering Conference (IECEC), AIAA-2015-3807, Orlando, FL, 2015.

[10] Wasserman, G. S., Reliability Verification, Testing and Analysis in Engineering Design, Marcal Dekker, Inc., New York, NY, 2003, pp. 203-239.

[11] Abernathy, R., The New Weibull Handbook, 5th. ed.,
SAE Publications, Warrendale, PA, 1993, pp. 6-1 - 6-23

[12] Smith, C. A. et al, "Goal Based Testing: A Risk Informed Process", Radioisotope Power Systems (RPS) Program report RPS-RPT-0104, September 2012.

\section{BIOGRAPHIES}

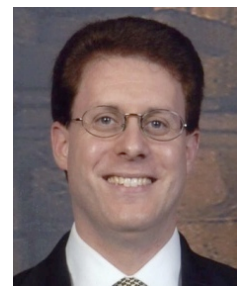

Kenneth Hibbard received his B.S. in Aerospace Engineering from the Pennsylvania State University, and his M.S. in Systems Engineering from the Johns Hopkins University. He spent eight years as a spacecraft systems and operations engineer at NASA Goddard working on the ACE, SOHO, and Swift spacecraft. At APL, Mr. Hibbard previously worked as the MESSENGER Deputy Mission Operations Manager, Formulation Deputy Project Systems Engineer for the Europa flagship mission study, the MSE for Implementation of the Precision Tracking Space System (a demonstration flight of APL-designed spacecraft hosting a new space-based sensor in support of MDA's Ballistic Missile Defense System), and as the Mission Systems Engineer for the Titan Mare Explorer (TiME) and Io Volcano Observer (IVO) Discovery proposals. $\mathrm{Mr}$. Hibbard is currently a principal systems engineer on multiple programs and proposals, including supporting the Stirling development efforts led by NASA GRC. He is also the Acting Deputy Program Development Manager at APL for Civil Space, and the Group Supervisor of APL's Space Systems Engineering Group.

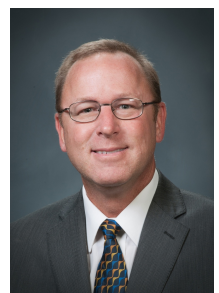

Mr. Mason is a nationally recognized expert in space power and propulsion systems with 28 years of professional experience at NASA Glenn Research Center (GRC) in Cleveland Ohio. His current position is Chief of the Thermal Energy Conversion Branch in the Power Division at GRC. His branch is responsible for the development of Stirling radioisotope generators and advanced reactor power systems for science and human exploration missions. The branch oversees the GRC Stirling Research Lab with 13 separate test stands that have accumulated over 800,000 operating hours on freepiston Stirling engines. Mr. Mason has written over 100 technical publications on space power and propulsion and generated several patent applications related to space nuclear power. He is a member of the American Institute of Aeronautics and Astronautics (AIAA) Nuclear and Future Flight Propulsion Technical Committee, the American Nuclear Society (ANS) Space Nuclear Committee, and the Interagency Advanced Power Group. Mr. Mason has been awarded the NASA Exceptional Achievement Medal (2006), the Rotary National Stellar Award (2010), and the NASA Outstanding Leadership Medal (2014). He holds a B.S. in Mechanical Engineering from the University of Dayton and a M.S. in Mechanical Engineering from Cleveland State University. 


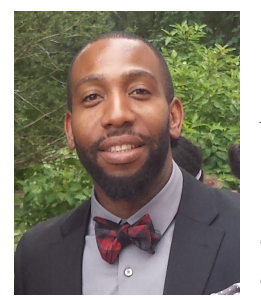

Obi Ndu received his B.S. and M.Eng. in Aerospace Engineering from University of Maryland, College Park. He has worked 14 years as an aerospace systems engineer in both commercial space and missile defense industries, covering commercial launch vehicles, commercial satellites, and weapons systems. His experience includes work on NASA GSFC's James Webb Space Telescope (JWST), Magnetospheric Multi-scale (MMS) Mission, Geostationary Operational Environmental Satellites $-R$ Series (GOES-R), the Van Allen Probes, and the MDA's Aegis Ballistic Missile Defense System Standard Missile. Currently, Mr. Ndu is a systems engineer in the Space Systems Engineering Group at APL and a Ph.D. candidate at UMCP's Department of Mechanical Engineering. His current work and research include modeling of complex system reliability using physicsbased degradation processes, quantification of dissimilarity between complex systems, and using Bayesian inference techniques to estimate the reliability of systems in limited design and operational data scenarios.

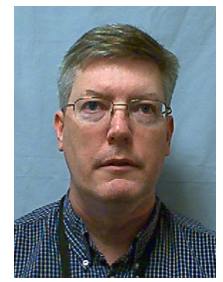

Dr. Smith has over 25 years of experience analyzing systems from risk, reliability, and safety perspectives. These systems included: NASA and DoD missions, payloads, ground communication systems, air traffic control systems, and missile systems. He is developing approaches to assess intentional threats against space assets using PRA and Game Theory techniques. He created and managed NASA's International Space Station Program Probabilistic Risk Assessment specifically geared toward quantifying the safety risk during operations. Dr. Smith is currently the reliability engineering lead for APL's Solar Probe Plus mission. He received his B.S. in Aerospace Engineering, M.S. in Engineering Management, and Ph.D. in Reliability Engineering all from the University of Maryland.

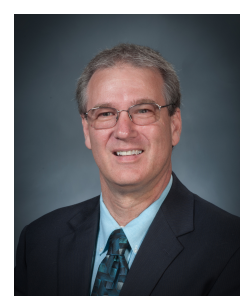

Mr. Withrow received his B.S. in Electrical Engineering from Ohio Northern University. Mr. Withrow has worked at NASA Lewis/Glenn Research Center for 28 years. He spent six year working in power systems technology development for the International Space Station, creating a distributed control system using Ada software and power electronics for the test facilities. Mr. Withrow supported the conversion of a commercial DC-9 aircraft into a microgravity research platform, and as the NASA Glenn Research Center (GRC) microgravity facility manager and test director, he coordinated and flew on over 400 microgravity research flights with over 18,000 parabolas on six different aircraft over thirteen years. During that period, he directly supported hundreds of scientists and engineers, and designed many microgravity experiments. Mr. Withrow was the lead electrical engineer for the 2.2 and 5.2 second drop towers at GRC for a six-year period. Mr. Withrow was the project manager for the Cold Gas Reaction Control System being designed for the Orion Ascent Abort-1 vehicle tests, along with supporting the Orion Pad Abort1 test crew module flight. Mr. Withrow is presently the Stirling Cycle Technology Development Manager at NASA GRC, supporting the development of Stirling technology for five years. Mr. Withrow supported the DOE as the Advanced Stirling Radioisotope Generator Deputy Project Manager for two years. Mr. Withrow has been awarded the Silver Snoopy (2007) and the Exceptional Achievement Medal (2013). In Mr. Withrow's free time, he is a Firefighter and EMT on his home community's fire department. 